\title{
NEW ELECTRICAL CHARACTERIZATION OF THE METAL-SEMICONDUCTOR INTERFACE IN GaAs SCHOTTKY JUNCTIONS
}

\author{
Zs.J. HORVÁTH \\ Research Institute for Technical Physics, Hungarian Academy of Sciences, Budapest, \\ P.O.Box 76, H-1325, Hungary \\ (Received August 8, 1990)
}

\begin{abstract}
The new possibilities of the electrical characterization of the metal-semiconductor interface in Schottky junctions are briefly outlined and demonstrated by using an example of experimental results taken from the literature. The interface parameters obtained for GaAs Schottky junctions with different metallizations are summarized.
\end{abstract}

PACS numbers: $73.30 .+y$

\section{Introduction}

Although the rectifying metal-semiconductor (Schottky) junctions are apparently rather simple structures, even their electrical behaviours have not been enough understood yet. Lately great efforts have been made to make clear the effect of the energy distribution of interface states on the barrier height $[1,2]$. However, the depth distribution of the interface states has at least as important role in the Schottky barrier formation as their energy distribution.

The energy relations and electrical characteristics may be described fairly well on the basis of the interfacial layer model of these junctions. This model assumes the presence of an interfacial layer and interface states at the metal-semiconductor (M-S) interface. It was originally assumed that the interfacial layer has insulating properties [3]. However, on the one hand, nowadays Schottky junctions without any insulating interfacial layer may be probably prepared (e.g. by MBE or silicide formation) and, on the other hand, the presence of an insulating layer at the $\mathrm{M}-\mathrm{S}$ interface is not necessary for the Fermi-level pinning [4]. The interfacial layer model is suitable to such structures as well. In these cases the thickness of the interfacial 
layer may be considered as the depth of the interface charge centroid from the $\mathrm{M}-\mathrm{S}$ interface.

Recently an evaluation method based on the interfacial layer model, has been proposed for the extraction of the interface state energy distribution and of the relative interfacial layer thickness (the ratio of the thickness of the interfacial layer to its relative dielectric constant) from the Schottky $I-V$ characteristics [5]. Further on a general expression for the equilibrium (zero bias) Schottky barrier height has also been derived [6]. This expression has no initial assumptions concerning the energy distribution of the interface states and the equilibrium interface charge, in contrast to the previous expressions $[7,8]$. Moreover, it describes the interface by using two parameters only: by the relative interfacial layer thickness and the equilibrium interface charge.

This evaluation method and the general expression give new possibilities for the characterization of the interface, and for obtaining relations between the interface parameters and the Schottky barrier height. If substituting the relative interfacial layer thickness obtained from the $I-V$ characteristics into the general expression, the equilibrium interface charge which is the only variable in this case, may also be obtained.

Using these possibilities, the first relations between the experimental barrier height, the relative interfacial layer thickness and interface charge values, and the interface state energy distribution spectra (demonstrating the validity of the interfacial layer model) has been obtained in $n$-type Au-GaAs junctions as a function of the annealing temperature [6]. It was found that the dependence of the equilibrium interface charge on the annealing temperature was in very good agreement with that of the interface state energy distribution spectra. These results indicated that the positive equilibrium interface charge responsible for the actual equilibrium barrier height values, was due to the ionization of donor-type interface states situated in the upper half of the forbidden gap. The near-ohmic behaviour of the characteristics after heat treatments at and above $350^{\circ} \mathrm{C}$, also might be explained by a significant increase of the density of these states (details see [6]).

In this paper the above possibilities (except for the evaluation of the interface state energy distribution spectrum) are demonstrated by using the experimental $I-V$ results obtained on Ti-GaAs Schottky junctions with different surface treatment by Meglicki and co-workers [9]. The relative interfacial layer thicknesses and equilibrium interface charges obtained for GaAs Schottky junctions with different metallizations are summarized.

\section{Results and discussion}

Recently Meglicki and co-workers [9] studied the reverse $I-V$ characteristics of Ti-GaAs Schottky contacts prepared by using three different surface passivation procedure: sample labelled $A$ had native oxide before the evaporation of the Schottky contact; from the surface of sample $B$ the native oxide was removed by consecutive etching in $\mathrm{H}_{2} \mathrm{O}_{2}$ and $\mathrm{HCl}$ before evaporation, while sample $\mathrm{C}$ underwent photochemical etching. It was found that both the saturation current and the 
slope of the reverse $I-V$ characteristics were different for these structures: sample A had the lowest saturation current and slope, while sample B had the highest current level and slope; sample $\mathrm{C}$ had intermediate characteristics.

The present author has evaluated the equilibrium barrier heights and the relative interfacial layer thicknesses from the reverse $I-V$ characteristics presented for the three examined samiples in [9]. As the forward $I-V$ characteristics were not known, the relative interfacial layer thickness has been evaluated by using the assumption that the interface state charge does not depend on the reverse bias (Model B [5]). Using the obtained data, the equilibrium interface charge has also been calculated by using the general expression mentioned above. The results are presented in Table I.

\section{TABLE I}

The equilibrium barrier height, relative interfacial layer thickness and equilibrium interface charge values evaluated from the reverse $I-V$ characteristics of Ti-GaAs Schottky junctions with different surface treatment before metallization [9].

\begin{tabular}{l|l|c|c|c}
\hline \hline Sample & Treatment & $\phi_{\text {bo }}[\mathrm{V}]$ & $\delta / \varepsilon_{\mathrm{i}}[\mathrm{nm}]$ & $Q_{\text {io }} \times 10^{-13}\left[\mathrm{C} / \mathrm{cm}^{2}\right]$ \\
\hline $\mathrm{A}$ & native & 0.77 & $0.20 \pm 0.02$ & $-1.42 \pm 0.15$ \\
$\mathrm{~B}$ & $\mathrm{I}_{2} \mathrm{O}_{2}+\mathrm{IICl}$ & 0.74 & $0.20 \pm 0.02$ & $-1.35 \pm 0.15$ \\
$\mathrm{C}$ & photochem. & 0.75 & $0.16 \pm 0.02$ & $-1.73 \pm 0.20$
\end{tabular}

It is seen that the barrier height decreased after the etching in $\mathrm{II}_{2} \mathrm{O}_{2}$ and $\mathrm{IICl}$, but the relative interfacial layer thickness did not decreased. The change of the barrier height was due to the decrease of the equilibrium interface charge. After the photochemical etching the relative interfacial layer thickness decreased, while the equilibrium interface charge increased resulting in a slight increase of the barrier height. It was considered by Meglicki and co-workers that the photochemical etching results in a $5 \mathrm{~nm}$-thick film of elemental arsenic at the GaAs surface. The relatively considerable change of the relative interfacial layer thickness and equilibrium interface charge may be connected with the formation of this film. On the other hand, Meglicki and co-workers concluded by using Levenburg-Marquardt $X^{2}$ analysis that the characteristics of sample $B$ are qualitatively different than those of samples $\mathrm{A}$ and $\mathrm{B}$. The results presented here may not confirm this statement: the interface parameters for sample $\mathrm{C}$ are different from those for sample $\mathrm{A}$ and B which are close to each other.

Table II summarizes the barrier height, relative interfacial layer thickness and equilibrium interface charge values evaluated from the $I-V$ characteristics obtained in unannealed GaAs Schottky junctions with different metallization by several authors. The lowest relative interfacial layer thicknesses were obtained for $\mathrm{Ti}$ [9]. Cr has an intermediate value [5]. Al [10] and $\mathrm{Au} \mathrm{[6]} \mathrm{gave} \mathrm{the} \mathrm{highest} \mathrm{ones.}$ The equilibrium interface charge values are in good correlation with the metal work functions which are also presented. This correlation is connected with the fact that except for Al metallization the Fermi level is pinned practically at the same energy position at the semiconductor surface for all the examined junctions. 
TABLE II

The metal work function, equilibrium barrier height, relative interfacial layer thickness and equilibrium interface charge values for GaAs Schottky junctions with different metallization.

\begin{tabular}{|c|c|c|c|c|}
\hline Metal & $\overline{\phi_{m}[\mathrm{~V}]}$ & $\overline{\phi_{\mathrm{bo}}[\mathrm{V}]}$ & $\delta / \varepsilon_{\mathrm{i}}[\mathrm{nm}]$ & $Q_{\text {io }} \times 10^{-13}\left[\mathrm{C} / \mathrm{cm}^{2}\right]$ \\
\hline $\mathrm{Al}^{1}$ & 4.28 & $0.55^{5}$ & 0.33 & -1.0 \\
\hline $\mathrm{Ti}^{2}$ & 4.33 & $0.74-0.77$ & $0.16-0.20$ & $-(1.3-1.7)$ \\
\hline $\mathrm{Cr}^{3}$ & 4.5 & 0.73 & 0.28 & -0.6 \\
\hline $\mathrm{Au}^{4}$ & 5.1 & 0.77 & 0.33 & +0.3 \\
\hline \multicolumn{5}{|c|}{$\begin{array}{l}{ }^{1} \text { UHV cleaved [10] } \\
{ }^{2} \text { etched (see Table I) [9] } \\
{ }^{3} \text { etched [5] } \\
{ }^{4} \text { etched [6] }\end{array}$} \\
\hline
\end{tabular}

\section{References}

[1] W. Mönch, J. Vac. Sci. Technol. B, Microelectron. Process. Phenom. 6, 1270 (1988).

[2] S.G. Anderson, C.M. Aldao, G.D. Waddil, I.M. Vitomorov, C. Capasso, J.H. Weaver, Appl. Phys. Lett. 55, 2547 (1989).

[3] J. Bardeen, Phys. Rev. 71, 717 (1947).

[4] G.N. Lu, C. Barret, T. Neffati, Rev. Phys. Appl. 22, 1169 (1987) (in French).

[5] Zs.J. Horváth, J. Appl. Phys. 63, 976 (1988).

[6] Zs.J. Horváth, Appl. Phys. Lett. 54, 931 (1989).

[7] A.M. Cowley, S.M. Sze, J. Appl. Phys. 36, 3212 (1965).

[8] J. Szatkowski, K. Sieranski, Solid-State Electron. 31, 257 (1988).

[9] Z. Meglicki, B.D. Nener, K. Prasad, H. Sharda, L. Faraone, A.G. Nassibian, Jpn. J. Appl. Phys. 27, L290 (1988).

[10] N. Newman, W.E. Spicer, E.R. Weber, J. Vac. Sci. Technol. B 5, 1020 (1987). 